\title{
An Elementary Proof of the Fritz-John and Karush-Kuhn- Tucker Conditions in Nonlinear Programming
}

\author{
S.I. Birbil, J. B. G. Frenk and G. J. Still
}

\begin{tabular}{|l|l|}
\hline \multicolumn{2}{|l|}{ ERIM REPORT SERIES RESEARCH IN MANAGEMENT } \\
\hline ERIM Report Series reference number & ERS-2005-057-LIS \\
\hline Publication & October 2005 \\
\hline Number of pages & 7 \\
\hline Persistent paper URL & \\
\hline Email address corresponding author & frenk@few.eur.nl \\
\hline Address & Erasmus Research Institute of Management (ERIM) \\
& RSM Erasmus University / Erasmus School of Economics \\
& Erasmus Universiteit Rotterdam \\
& P.O.Box 1738 \\
& 3000 DR Rotterdam, The Netherlands \\
& Phone: + 31 10 408 1182 \\
& Fax: + 31 10 408 9640 \\
& Email: info@erim.eur.nl \\
& Internet: $\quad$ www.erim.eur.nl \\
\hline
\end{tabular}

Bibliographic data and classifications of all the ERIM reports are also available on the ERIM website: www.erim.eur.nl 


\section{ERASMUS RESEARCH INSTITUTE OF MANAGEMENT}

\section{REPORT SERIES}

\section{RESEARCH IN MANAGEMENT}

\begin{tabular}{|l|l|}
\hline \multicolumn{2}{|l|}{ ABSTRACT AND KEYWORDS } \\
\hline Abstract & $\begin{array}{l}\text { In this note we give an elementary proof of the Fritz-John and Karush-Kuhn-Tucker conditions } \\
\text { for nonlinear finite dimensional programming problems with equality and/or inequality } \\
\text { constraints. The proof avoids the implicit function theorem usually applied when dealing with } \\
\text { equality constraints and uses a generalization of Farkas lemma and the Bolzano-Weierstrass } \\
\text { property for compact sets. }\end{array}$ \\
\hline Free Keywords & $\begin{array}{l}\text { Nonlinear programming, Fritz-John conditions, Karush-Kuhn-Tucker conditions } \\
\text { Availability } \\
\text { The ERIM Report Series is distributed through the following platforms: } \\
\text { Academic Repository at Erasmus University (DEAR), DEAR ERIM Series Portal } \\
\text { Social Science Research Network (SSRN), SSRN ERIM Series Webpage } \\
\text { Research Papers in Economics (REPEC), REPEC ERIM Series Webpage }\end{array}$ \\
\hline Classifications & $\begin{array}{l}\text { The electronic versions of the papers in the ERIM report Series contain bibliographic metadata } \\
\text { by the following classification systems: } \\
\text { Library of Congress Classification, (LCC) LCC Webpage } \\
\text { Journal of Economic Literature, (JEL), JEL Webpage } \\
\text { ACM Computing Classification System CCS Webpage } \\
\text { Inspec Classification scheme (ICS), ICS Webpage }\end{array}$ \\
\hline
\end{tabular}




\title{
An Elementary Proof of the Fritz-John and Karush-Kuhn-Tucker Conditions in Nonlinear Programming
}

\author{
Ş. İ. Birbil ${ }^{\dagger}$, J. B. G. Frenk ${ }^{\ddagger *}$ and G. J. Still ${ }^{\natural}$ \\ ${ }^{\dagger}$ Faculty of Engineering and Natural Sciences, Sabancı University \\ Orhanli-Tuzla, 34956, Istanbul, Turkey. \\ sibirbilesabanciuniv.edu \\ ${ }^{\ddagger}$ Econometric Institute, Erasmus University Rotterdam \\ P.O. Box 1738, 3000 DR Rotterdam, The Netherlands. \\ frenk@few.eur.nl \\ ${ }^{\natural}$ Department of Mathematical Sciences, University of Twente \\ P.O. Box 217, 7500 AE Enschede, The Netherlands. \\ g.still@math.twente.nl
}

\begin{abstract}
In this note we give an elementary proof of the Fritz-John and Karush-Kuhn-Tucker conditions for nonlinear finite dimensional programming problems with equality and/or inequality constraints. The proof avoids the implicit function theorem usually applied when dealing with equality constraints and uses a generalization of Farkas lemma and the Bolzano-Weierstrass property for compact sets.
\end{abstract}

Keywords. Nonlinear programming. Fritz-John conditions, Karush-Kuhn-Tucker conditions

\section{Introduction}

Let A be an $m \times n$ matrix with rows $\mathbf{a}_{k}^{\top}, 1 \leq k \leq m, \mathbf{b} \in \mathbb{R}^{m}$ an $m$-dimensional vector, and $f_{i}: \mathbb{R}^{n} \rightarrow \mathbb{R}$, $0 \leq i \leq q$ some non-affine, continuously differentiable functions. We consider the optimization problem

$$
\min \left\{f_{0}(\mathbf{x}): \mathbf{x} \in \mathcal{F}_{P}\right\}, \mathcal{F}_{P}:=\left\{\mathbf{x} \in \mathbb{R}^{n}: \mathbf{a}_{k}^{\top} \mathbf{x} \leq b_{k}, 1 \leq k \leq m, f_{i}(\mathbf{x}) \leq 0,1 \leq i \leq q\right\},
$$

and the program including equalities

$$
\min \left\{f_{0}(\mathbf{x}): \mathbf{x} \in \mathcal{F}_{Q}\right\}, \mathcal{F}_{Q}:=\mathcal{F}_{P} \cap\left\{\mathbf{x} \in \mathbb{R}^{n}: h_{j}(\mathbf{x})=0,1 \leq j \leq r\right\}
$$

where the functions $h_{j}: \mathbb{R}^{n} \rightarrow \mathbb{R}, 1 \leq j \leq r$, are non-affine and continuously differentiable.

Two basic results covered in every course on nonlinear programming are the Fritz-John (FJ) and Karush-Kuhn-Tucker (KKT) necessary conditions for the local minimizers of optimization problems (P) and (Q) [7-9]. Denoting the nonnegative orthant of $\mathbb{R}^{l}$ by $\mathbb{R}_{+}^{l}$, the FJ necessary conditions for problem

\footnotetext{
${ }^{*}$ Corresponding author.
} 
(P) are given by the following: If $\mathrm{x}_{P}$ is a local minimizer of problem $(\mathrm{P})$, then there exist (see for example $[2,5])$ vectors $\mathbf{0} \neq \lambda \in \mathbb{R}_{+}^{q+1}$ and $\nu \in \mathbb{R}_{+}^{m}$ satisfying

$$
\begin{aligned}
& \sum_{i=0}^{q} \lambda_{i} \nabla f_{i}\left(\mathbf{x}_{P}\right)+\sum_{k=1}^{m} \nu_{k} \mathbf{a}_{k}=\mathbf{0} \\
& \lambda_{i} f_{i}\left(\mathbf{x}_{P}\right)=0,1 \leq i \leq q \text { and } \nu_{k}\left(\mathbf{a}_{k}^{\top} \mathbf{x}_{P}-b_{k}\right)=0,1 \leq k \leq m .
\end{aligned}
$$

For optimization problem (Q) the resulting FJ conditions are as follows: If $\mathbf{x}_{Q}$ is a local minimizer of problem $(\mathrm{Q})$, then there exist (see for example $[2,5]$ ) vectors $(\lambda, \nu) \in \mathbb{R}_{+}^{q+1+m}, \mu \in \mathbb{R}^{r}$ with $(\lambda, \mu) \neq \mathbf{0}$ satisfying

$$
\begin{aligned}
& \sum_{i=0}^{q} \lambda_{i} \nabla f_{i}\left(\mathbf{x}_{Q}\right)+\sum_{j=1}^{r} \mu_{j} \nabla h_{j}\left(\mathbf{x}_{Q}\right)+\sum_{k=1}^{m} \nu_{k} \mathbf{a}_{k}=\mathbf{0}, \\
& \lambda_{i} f_{i}\left(\mathbf{x}_{Q}\right)=0,1 \leq i \leq q \text { and } \nu_{k}\left(\mathbf{a}_{k}^{\top} \mathbf{x}_{Q}-b_{k}\right)=0,1 \leq k \leq m .
\end{aligned}
$$

If $\lambda_{0}$ given in conditions (FJP) and (FJQ) can be chosen positive, then the resulting necessary conditions are called the KKT conditions for problems (P) and (Q), respectively. A sufficient condition for $\lambda_{0}$ to be positive is given by a so-called first-order constraint qualification. In the next section we firstly give an elementary proof of the FJ and KKT conditions for problem (P). Then the same proof is given for optimization problem (Q) by using a perturbation argument but avoiding the implicit function theorem.

\section{The FJ and KKT conditions for problems (P) and (Q)}

For $\delta>0$ and $\overline{\mathbf{x}} \in \mathbb{R}^{n}$, let $\mathcal{N}(\overline{\mathbf{x}}, \delta)$ denote a $\delta$-neighborhood of $\overline{\mathbf{x}}$ given by

$$
\mathcal{N}(\overline{\mathbf{x}}, \delta):=\left\{\mathbf{x} \in \mathbb{R}^{n}:\|\mathbf{x}-\overline{\mathbf{x}}\| \leq \delta\right\}
$$

A vector $\mathbf{x}_{P}$ is called a local minimizer of optimization problem (P) (respectively, for optimization problem (Q) if $\mathbf{x}_{P} \in \mathcal{F}_{P}$ (respectively, $\left.\mathbf{x}_{P} \in \mathcal{F}_{Q}\right)$ and there exists some $\delta>0$ such that $f_{0}\left(\mathbf{x}_{P}\right) \leq f_{0}(\mathbf{x})$ for every $\mathbf{x} \in \mathcal{F}_{P} \cap \mathcal{N}\left(\mathbf{x}_{P}, \delta\right)$ (respectively, $\mathbf{x} \in \mathcal{F}_{Q} \cap \mathcal{N}\left(\mathbf{x}_{P}, \delta\right)$ ).

We introduce the active index sets $I(\mathbf{x}):=\left\{1 \leq i \leq q: f_{i}(\mathbf{x})=0\right\}$ and $K(\mathbf{x})=\{1 \leq k \leq m:$ $\left.\mathbf{a}_{k}^{\top} \mathbf{x}=b_{k}\right\}$, and denote by $B(\mathbf{x})$, the matrix consisting of the corresponding active rows $\mathbf{a}_{k}^{\top}, k \in K(\mathbf{x})$.

Lemma 2.1 If $\mathbf{x}_{P}$ is a local minimizer of problem $(P)$, then $\max \left\{\nabla f_{i}\left(\mathbf{x}_{P}\right)^{\top} \mathbf{d}: i \in I\left(\mathbf{x}_{P}\right) \cup\{0\}\right\} \geq 0$ for every $\mathbf{d}$ such that $B\left(\mathbf{x}_{P}\right) \mathbf{d} \leq \mathbf{0}$.

Proof. Suppose by contradiction there exists some $\mathbf{d}_{0}$ satisfying $B\left(\mathbf{x}_{P}\right) \mathbf{d}_{0} \leq \mathbf{0}$ and

$$
0>\nabla f_{i}\left(\mathbf{x}_{P}\right)^{\top} \mathbf{d}_{0}=\lim _{t \downarrow 0} \frac{f_{i}\left(\mathbf{x}_{P}+t \mathbf{d}_{0}\right)-f_{i}\left(\mathbf{x}_{P}\right)}{t}
$$

for every $i \in I\left(\mathbf{x}_{P}\right) \cup\{0\}$. By the finiteness of the sets $\{0, \ldots, q\}$ and $\{1, \ldots, m\}$ and the continuity of $f_{i}$ this implies the existence of some $t_{0}>0$ satisfying

$$
f_{i}\left(\mathbf{x}_{P}+t \mathbf{d}_{0}\right)<0, i \notin I\left(\mathbf{x}_{P}\right), f_{i}\left(\mathbf{x}_{P}+t \mathbf{d}_{0}\right)<f_{i}\left(\mathbf{x}_{P}\right), i \in I\left(\mathbf{x}_{P}\right) \cup\{0\}, A\left(\mathbf{x}_{P}+t \mathbf{d}_{0}\right) \leq \mathbf{b}
$$

for every $0<t \leq t_{0}$. Hence the vector $\mathbf{x}_{P}+t \mathbf{d}_{0}$ belongs to $\mathcal{F}_{P}$ and satisfies $f_{0}\left(\mathbf{x}_{P}+t \mathbf{d}_{0}\right)<f_{0}\left(\mathbf{x}_{P}\right)$ for every $0<t \leq t_{0}$. This contradicts that $\mathbf{x}_{P}$ is a local minimum.

Remark 2.1 If the function $f_{0}$ is pseudo-convex and the functions $f_{i}, 1 \leq i \leq q$ are strictly pseudoconvex, then for a feasible $\mathbf{x}_{P}$ the reverse implication in Lemma 2.1 also holds and in this result local 
minimizer is replaced by global minimizer. A proof of this will be given at the end of this section. Moreover, if $\max _{B \mathbf{d} \leq \mathbf{0},\|\mathbf{d}\|=1}\left\{\nabla f_{i}\left(\mathbf{x}_{P}\right)^{\top} \mathbf{d}: i \in I\left(\mathbf{x}_{P}\right) \cup\{0\}\right\}>0$ and $\mathbf{x}_{P}$ feasible, then one can show that $\mathbf{x}_{P}$ is a local minimum of order one ([5]), i.e., there exists some $\delta>0$ and $c>0$ such that $f_{0}(\mathbf{x})-f_{0}\left(\mathbf{x}_{P}\right) \geq c\left\|\mathbf{x}-\mathbf{x}_{P}\right\|$ for every $\mathbf{x} \in \mathcal{F}_{P} \cap \mathcal{N}\left(\mathbf{x}_{P}, \delta\right)$.

The proof of the $F J$ conditions for problem (P) will be based on the following generalization of Farkas lemma ([6]). For completeness, a short proof, using the strong duality result for linear programming, will be given in the appendix.

Lemma 2.2 Let $\Delta_{s} \subseteq \mathbb{R}_{+}^{s}$ be the unit simplex. If $B$ is a $p \times n$ matrix and $\mathbf{c}_{i} \in \mathbb{R}^{n}, 1 \leq i \leq s$, some given vectors, then the following conditions are equivalent:

1. For every $\mathbf{d} \in \mathbb{R}^{n}$ satisfying $B \mathbf{d} \leq \mathbf{0}$ it holds that $\max _{1 \leq i \leq s} \mathbf{c}_{i}^{\top} \mathbf{d} \geq 0$.

2. There exists some $\lambda \in \Delta_{s}$ and $\mu \in \mathbb{R}_{+}^{p}$ satisfying $\sum_{i=1}^{s} \lambda_{i} \mathbf{c}_{i}+B^{\top} \mu=\mathbf{0}$.

Proof. (FJ conditions for problem (P)) By combining Lemma 2.1 and Lemma 2.2, the FJ conditions follow.

It is well-known that the KKT conditions follow from the FJ conditions under some constraint qualification. We say that the Mangasarian-Fromovitz (MF) constraint qualification for problem (P) holds at a feasible point $\mathbf{x}$ if there exists some $\mathbf{d}_{0}$ satisfying

$$
B(\mathbf{x}) \mathbf{d}_{0} \leq 0 \text { and } \max _{i \in I(\mathbf{x})}\left\{\nabla f_{i}(\mathbf{x})^{\top} \mathbf{d}_{0}\right\}<0
$$

We now show that at a local minimizer $\mathbf{x}_{P}$ of problem $(\mathrm{P})$ satisfying the MF constraint qualification, the KKT conditions must hold.

Proof. (KKT conditions for problem (P)). Assume that $\lambda_{0}=0$ in the FJ conditions. Applying Lemma 2.2 to the FJ conditions with $\lambda_{0}=0$ we obtain that $\max _{i \in I\left(\mathbf{x}_{P}\right)} \nabla f_{i}\left(\mathbf{x}_{P}\right)^{\top} \mathbf{d} \geq 0$ for every $B\left(\mathbf{x}_{P}\right) \mathbf{d} \leq \mathbf{0}$. This contradicts the MF constraint qualification.

To prove the FJ and KKT conditions for problem (Q) without using the implicit function theorem we consider for a local minimizer $\mathbf{x}_{Q}$ of problem $(\mathrm{Q})$ and $\delta>0$ appropriately chosen and $\epsilon>0$, the perturbed feasible region

$$
\mathcal{F}_{\delta}(\epsilon):=\mathcal{F}_{P} \cap \mathcal{N}\left(\mathbf{x}_{Q}, \delta\right) \cap\left\{\mathbf{x} \in \mathbb{R}^{n}: h_{j}(\mathbf{x}) \leq \epsilon,-h_{j}(\mathbf{x}) \leq \epsilon, 1 \leq j \leq r\right\}
$$

and the associated optimization problem

$$
\min \left\{f_{0}(\mathbf{x})+\left\|\mathbf{x}-\mathbf{x}_{Q}\right\|^{2}: \mathbf{x} \in \mathcal{F}_{\delta}(\epsilon)\right\}
$$

Since the feasible region is compact a global minimizer $\mathbf{x}_{Q}(\epsilon)$ exists for problem $\left(Q_{\delta}(\epsilon)\right)$. For these global minimizers one can show the following result.

Lemma 2.3 For any sequence $\epsilon_{l} \downarrow 0$ it follows that $\lim _{l \uparrow \infty} \mathbf{x}_{Q}\left(\epsilon_{l}\right)=\mathbf{x}_{Q}$.

Proof. Let us assume to the contrary that there exists a sequence $\mathbf{x}_{Q}\left(\epsilon_{l}\right), l \in \mathbb{N}$ which does not converge to $\mathbf{x}_{Q}$. By $\left\|\mathbf{x}_{Q}\left(\epsilon_{l}\right)-\mathbf{x}_{Q}\right\| \leq \delta$ and the Bolzano-Weierstrass property for compact sets there exists some subsequence $\mathbf{x}_{Q}\left(\epsilon_{l}\right), l \in L \subseteq \mathbb{N}$ satisfying

$$
\lim _{l \uparrow \infty, l \in L} \mathbf{x}_{Q}\left(\epsilon_{l}\right)=\overline{\mathbf{x}} \neq \mathbf{x}_{Q}
$$


By continuity $\overline{\mathbf{x}}$ must be feasible for problem $(\mathrm{Q})$. Since $\mathbf{x}_{Q}$ is feasible for $\left(Q_{\delta}\left(\epsilon_{l}\right)\right), l \in L$ it follows that

$$
f_{0}\left(\mathbf{x}_{Q}\left(\epsilon_{l}\right)\right)+\left\|\mathbf{x}_{Q}\left(\epsilon_{l}\right)-\mathbf{x}_{Q}\right\|^{2} \leq f_{0}\left(\mathbf{x}_{Q}\right)
$$

for every $l \in L$. Taking now the limit in relation (2.2) we find by relation (2.1) that

$$
f_{0}(\overline{\mathbf{x}})+\left\|\overline{\mathbf{x}}-\mathbf{x}_{Q}\right\|^{2} \leq f_{0}\left(\mathbf{x}_{Q}\right)
$$

and this contradicts the local optimality of $\mathbf{x}_{Q}$ for problem (Q).

If $\mathbf{x}_{Q}$ is a strict local minimizer, i.e $f_{0}\left(\mathbf{x}_{Q}\right)<f_{0}(\mathbf{x})$ for every $\mathbf{x} \in \mathcal{F}_{Q} \cap \mathcal{N}\left(\mathbf{x}_{Q}, \delta\right)$, we do not need in the above proof the penalty term $\left\|\mathbf{x}-\mathbf{x}_{Q}\right\|^{2}$. Using Lemma 2.3 one can now give an elementary proof of the FJ and KKT conditions for a local minimizer $\mathbf{x}_{Q}$ of problem $(\mathrm{Q})$.

Proof. (FJ conditions for problem (Q)) Let $\epsilon_{l}$ be a strictly decreasing sequence and consider the associated optimal solutions $x_{Q}\left(\epsilon_{l}\right)$ of $\left(Q_{\delta}\left(\epsilon_{l}\right)\right)$. For notational convenience we denote $x_{Q}\left(\epsilon_{l}\right)$ by $\mathbf{x}^{(l)}$ and by Lemma 2.3 there exists some $l \geq l_{0}$ such that $\left\|\mathbf{x}^{(l)}-\mathbf{x}_{Q}\right\|<\delta$ for every $l \geq l_{0}$. Introduce now the set

$$
J_{l}:=\left\{1 \leq j \leq r: h_{j}\left(\mathbf{x}^{(l)}\right)=\epsilon_{l} \text { or } h_{j}\left(\mathbf{x}^{(l)}\right)=-\epsilon_{l}\right\} .
$$

The set of all subsets of the finite set $\{1, \ldots, r\}$ is finite and so the sequence $J_{l}, l \in \mathbb{N}$ contains some subset $\bar{J} \subseteq\{1, \ldots, r\}$ such that $L:=\left\{l \in \mathbb{N}: J_{l}=\bar{J}\right\}$ is infinite. Applying now for every $l \geq l_{0}$ and $l \in L$ the FJ conditions to problem $\left(Q_{\delta}\left(\epsilon_{l}\right)\right)$ we obtain that there exist vectors $\lambda_{l} \in \mathbb{R}_{+}^{q+1}, \mu_{l} \in \mathbb{R}^{|\bar{J}|}, \nu_{l} \in \mathbb{R}_{+}^{m}$, $\mathbf{0} \neq\left(\lambda_{l}, \mu_{l}\right)$, satisfying

$$
\begin{array}{r}
-\lambda_{0 l} \mathbf{g}\left(\mathbf{x}^{(l)}\right)-\sum_{i=1}^{q} \lambda_{i l} \nabla f_{i}\left(\mathbf{x}^{(l)}\right)-\sum_{j \in \bar{J}} \mu_{j l} \nabla h_{j}\left(\mathbf{x}^{(l)}\right)=\sum_{k=1}^{m} \nu_{k l} \mathbf{a}_{k} \\
\nu_{k l}\left(\mathbf{a}_{k}^{\top} \mathbf{x}^{(l)}-b_{k}\right)=0,1 \leq k \leq m, \text { and } \lambda_{i l} f_{i}\left(\mathbf{x}^{(l)}\right)=0,1 \leq i \leq q .
\end{array}
$$

with $g(\mathbf{x}):=\nabla f_{0}(\mathbf{x})+2\left(\mathbf{x}-\mathbf{x}_{Q}\right)$. By relation (2.3) and Caratheodory's lemma (see Appendix) one can find for every $l \in L$ some subset $K_{l} \subseteq\{1, \ldots, m\}$ and a vector $\nu_{l}^{*} \in \mathbb{R}_{+}^{\left|K_{l}\right|}$ satisfying

$$
-\lambda_{0 l} \mathbf{g}\left(\mathbf{x}^{(l)}\right)-\sum_{i=1}^{q} \lambda_{i l} \nabla f_{i}\left(\mathbf{x}^{(l)}\right)-\sum_{j \in \bar{J}} \mu_{j l} \nabla h_{j}\left(\mathbf{x}^{(l)}\right)=\sum_{k \in K_{l}} \nu_{k l}^{*} \mathbf{a}_{k}
$$

and the vectors $\mathbf{a}_{k}, k \in K_{l}$ are linearly independent. Since $\mathbf{0} \neq\left(\lambda_{l}, \mu_{l}\right)$ we may assume in relation (2.4) that the vector $\left(\lambda_{l}, \mu_{l}, \nu_{l}^{*}\right)$ has Euclidean norm 1. Again by selecting an infinite subsequence $L_{0} \subseteq L$ if necessary we can assume $K_{l}=\bar{K}$ (the same) for all $l \in L_{0}$. By the Bolzano-Weierstrass theorem the sequence of vectors $\left(\lambda_{l}, \mu_{l}, \nu_{l}^{*}\right), l \in L_{0}$ has a converging subsequence, i.e, there exists an infinite set $L_{1} \subseteq L_{0}$ with $\lim _{l \in L_{1}, l \uparrow \infty}\left(\lambda_{l}, \mu_{l}, \nu_{l}^{*}\right)=(\bar{\lambda}, \bar{\mu}, \bar{\nu})$ and $(\bar{\lambda}, \bar{\mu}, \bar{\nu})$ having Euclidean norm 1. Moreover, it follows by Lemma 2.3 and the continuity of $h_{j}$ that $\bar{J} \subseteq\left\{1 \leq j \leq r: h_{j}\left(\mathbf{x}_{Q}\right)=0\right\}$. Applying again Lemma 2.3 and the continuity of the gradients the desired result follows from relation (2.4) by letting $l \in L_{1}$ converge to infinity leading to the $\mathrm{FJ}$ condition:

$$
\sum_{i=0}^{q} \bar{\lambda}_{i} \nabla f_{i}\left(\mathbf{x}_{Q}\right)+\sum_{j \in \bar{J}} \bar{\mu}_{j} \nabla h_{j}\left(\mathbf{x}_{Q}\right)+\sum_{k \in \bar{K}} \bar{\nu}_{k} \mathbf{a}_{k}=\mathbf{0} .
$$

By construction the vectors $\mathbf{a}_{k}, k \in \bar{K}$, are linearly independent. Since $(\bar{\lambda}, \bar{\mu}, \bar{\nu})$ has Euclidean norm 1 and $\mathbf{a}_{k}, k \in \bar{K}$, are linearly independent this implies $(\bar{\lambda}, \bar{\mu}) \neq \mathbf{0}$.

For problem $(\mathrm{Q})$ we introduce the following constraint qualification: The MF constraint qualification for problem $(\mathrm{Q})$ is said to hold at a feasible point $\mathrm{x}$ if

MF1. $\nabla h_{j}(\mathbf{x}), 1 \leq j \leq r$ are linearly independent. 
MF2. $\operatorname{lin}\left\{\nabla h_{j}(\mathbf{x}), 1 \leq j \leq r\right\} \cap \operatorname{lin}\left\{\mathbf{a}_{k}, k \in K(\mathbf{x})\right\}=\{\mathbf{0}\}$.

MF3. There exists some $\mathbf{d}_{0}$ satisfying

$$
B(\mathbf{x}) \mathbf{d}_{0} \leq \mathbf{0}, \nabla h_{j}(\mathbf{x})^{\top} \mathbf{d}_{0}=0,0 \leq j \leq r, \text { and } \max _{i \in I(\mathbf{x})}\left\{\nabla f_{i}(\mathbf{x})^{\top} \mathbf{d}_{0}\right\}<0 .
$$

This is a natural condition. Without condition (MF2) a FJ point need not be a KKT point as shown by the 2-dimensional optimization problem (with minimizer and FJ point $\mathbf{x}_{Q}=\mathbf{0}$ )

$$
\min \left\{x_{1}: x_{2} \leq 0,-x_{2} \leq 0, x_{2}-x_{1}^{2}=0\right\} .
$$

Proof. (KKT conditions for problem (Q)) To show that at a minimizer $\mathbf{x}_{Q}$ of problem (Q) satisfying the MF constraint qualification the KKT condition must hold we assume to the contrary that in the FJ condition for problem (Q) we have $\lambda_{0}=0$. By (MF3) it must follow that $\lambda=\mathbf{0}$ and using $(\lambda, \mu) \neq \mathbf{0}$ it follows that $\mu \neq \mathbf{0}$. Applying now (MF2) and (MF3) to the FJ conditions with $\lambda=\mathbf{0}$ and $\mu \neq \mathbf{0}$ we obtain a contradiction.

As observed in Remark 2.1 we will now show for $f_{0}$ pseudo-convex and $f_{i}, 1 \leq i \leq q$ strictly pseudoconvex on $\mathbb{R}^{n}$, that for $\mathbf{x}_{P} \in \mathcal{F}_{P}$ the condition $\max \left\{\nabla f_{i}\left(\mathbf{x}_{P}\right)^{\top} \mathbf{d}: i \in I\left(\mathbf{x}_{P}\right) \cup\{0\}\right\} \geq 0$ for every $\mathbf{d}$ such that $B\left(\mathbf{x}_{P}\right) \mathbf{d} \leq \mathbf{0}$ implies that $\mathbf{x}_{P}$ is an global minimizer of problem $(\mathrm{P})$. Recall that a function $\phi: \mathbb{R}^{n} \mapsto \mathbb{R}$ is called pseudo-convex on $\mathbb{R}^{n}$ if $\phi$ is differentiable on $\mathbb{R}^{n}$ and $\nabla \phi(\mathbf{x})^{\top} \mathbf{d} \geq 0$ implies $\phi(\mathbf{x}+\mathbf{d}) \geq \phi(\mathbf{x})$ for every $\mathbf{x}, \mathbf{d} \in \mathbb{R}^{n}$. It is called strictly pseudo-convex on $\mathbb{R}^{n}$ if $\phi$ is differentiable and $\nabla \phi(\mathbf{x})^{\top} \mathbf{d} \geq 0$ implies $\phi(\mathbf{x}+\mathbf{d})>\phi(\mathbf{x})$ for every $\mathbf{x} \in \mathbb{R}^{n}$ and $\mathbf{0} \neq \mathbf{d} \in \mathbb{R}^{n}[1]$.

Proof. (Converse of Lemma 2.1 for $f_{0}$ pseudo-convex and $f_{i}, 1 \leq i \leq q$ strictly pseudo-convex) To prove the converse of Lemma 2.1 let us assume by contradiction that the feasible $\mathbf{x}_{P}$ is not an global minimizer of problem $(\mathrm{P})$. Hence there exists some $\mathbf{x}_{0} \in \mathcal{F}_{P}$ satisfying $f_{0}\left(\mathbf{x}_{0}\right)<f_{0}\left(\mathbf{x}_{P}\right)$. By the pseudoconvexity of $f_{0}$ this implies that $\nabla f_{0}\left(\mathbf{x}_{P}\right)^{\top}\left(\mathbf{x}_{0}-\mathbf{x}_{P}\right)<0$. Also by strict pseudo-convexity of $f_{i}, 1 \leq$ $i \leq q$ using $f_{i}\left(\mathbf{x}_{0}\right) \leq 0=f_{i}\left(\mathbf{x}_{P}\right), i \in I\left(\mathbf{x}_{P}\right)$ and $\mathbf{x}_{0} \neq \mathbf{x}_{P}$ we obtain that $\nabla f_{i}\left(\mathbf{x}_{P}\right)^{\top}\left(\mathbf{x}_{0}-\mathbf{x}_{P}\right)<0$ for every $i \in I\left(\mathbf{x}_{P}\right)$. Finally it holds that $B\left(\mathbf{x}_{P}\right)\left(\mathbf{x}_{0}-\mathbf{x}_{P}\right) \leq \mathbf{0}$ and we arrive at a contradiction to our initial assumption.

Combining Lemma 2.1 and 2.2 we immediately obtain the following result ([2]).

Lemma 2.4 Let $f_{0}$ be pseudo-convex and $f_{i}, 1 \leq i \leq q$ strictly pseudo-convex. Then it follows that $\mathbf{x}_{P} \in \mathcal{F}_{P}$ is a global minimizer of $(P)$ if and only if $\mathbf{x}_{P}$ satisfies the FJ conditions.

\section{Conclusion.}

In this note we have shown that the basic results in nonlinear programming are a natural and direct consequence of basic results in linear programming and analysis. In our proof we could avoid the implicit function theorem usually applied in the proof of the FJ conditions for problem (Q) (see for example $[2,5])$. The proof of the implicit function theorem ([11]) and its understanding is in general difficult for undergraduate/graduate students in the applied computational sciences. This concern was also the main objective for constructing an alternative elementary proof by McShane ([10]) for the FJ and KKT conditions for problem (Q). By not regarding separately linear and nonlinear inequalities the result in [10] is weaker than ours (also the linear independence constraint qualification for (Q) is used) and his proof uses the penalty approach of nonlinear programming (see also [3] for a similar proof). As such this 
technique and the technique used in this paper have their pros and cons. An advantage of the presented approach for problem $(\mathrm{P})$ is the fact that it can easily identify the class of functions for which the FJ conditions for problem $(\mathrm{P})$ are not only necessary but also sufficient. This seems to be difficult to show by means of the penalty approach of McShane. However, to our belief the main advantage of our proof technique is its display of a natural connection between linear and nonlinear programming.

\section{Appendix.}

In this appendix we give a short proof of Lemma 2.2 by means of the strong duality theorem for linear programming.

Proof. To verify $1 \Rightarrow 2$ we observe that

$$
0=\min _{B \mathbf{d} \leq \mathbf{0}} \max _{1 \leq i \leq s} \mathbf{c}_{i}^{\top} \mathbf{d}=\min _{B \mathbf{d} \leq \mathbf{0}, \mathbf{c}_{i}^{\top} \mathbf{d}-z \leq 0,1 \leq i \leq s} z .
$$

This is a linear programming problem and by the strong duality theorem of linear programming (cf.[4]) we obtain

$$
\min _{B \mathbf{d} \leq \mathbf{0}, \mathbf{c}_{i}^{\top} \mathbf{d}-z \leq 0,1 \leq i \leq s} z=\max \left\{\mathbf{0}^{\top}\left(\begin{array}{l}
\lambda \\
\mu
\end{array}\right): \sum_{i=1}^{s} \lambda_{i} \mathbf{c}_{i}+B^{\top} \mu=\mathbf{0}, \lambda \in \Delta_{s}, \mu \in \mathbb{R}_{+}^{p}\right\} .
$$

Applying now relations (4.1) and (4.2) we know that the feasible region of the dual problem is not empty and so there exist some $\lambda \in \Delta_{p}$ and $\mu \in \mathbb{R}_{+}^{p}$ satisfying $\sum_{i=1}^{s} \lambda_{i} \mathbf{c}_{i}^{\top} \mathbf{d}+B^{\top} \mu=\mathbf{0}$. To show the reverse implication it follows that there exists some $\lambda \in \Delta_{s}$ and $\mu \in \mathbb{R}_{+}^{p}$ satisfying $\sum_{i=1}^{s} \lambda_{i} \mathbf{c}_{i}^{\top} \mathbf{d}=-\mu^{\top} B \mathbf{d}$ for every $\mathbf{d} \in \mathbb{R}^{n}$. Hence for $B \mathbf{d} \leq \mathbf{0}$ and using $\mu \in \mathbb{R}_{+}^{p}$ we obtain $\max _{1 \leq i \leq s} \mathbf{c}_{i}^{\top} \mathbf{d} \geq \sum_{i=1}^{s} \lambda_{i} \mathbf{c}_{i}^{\top} \mathbf{d} \geq 0$.

In our analysis we also use the following result known as Caratheodory's lemma.

Lemma 4.1 Let $\mathbf{v} \in \mathbb{R}^{m}$ be represented as cone combination $\mathbf{v}=\sum_{k=1}^{m} \nu_{k} \mathbf{a}_{k}, \nu_{k} \geq 0$. Then there is a representation $\mathbf{v}=\sum_{k \in \bar{K}} \bar{\nu}_{k} \mathbf{a}_{k}, \bar{\nu}_{k}>0, k \in \bar{K}$ such that $\mathbf{a}_{k}, k \in \bar{K}$ are linearly independent.

Proof. We can assume

$$
\mathbf{v}=\sum_{k=1}^{m} \nu_{k} \mathbf{a}_{k}, \text { with } \nu_{k}>0
$$

and suppose that the vectors $\mathbf{a}_{k}, k=1, \ldots, m$ are linearly dependent. So there is a non-trivial combination $\mathbf{0}=\sum_{k=1}^{m} \tau_{k} \mathbf{a}_{k}$. By multiplying this relation by a factor $\rho$ and adding to (4.3) we find

$$
\mathbf{v}=\sum_{k=1}^{m}\left(\nu_{k}+\rho \tau_{k}\right) \mathbf{a}_{k}
$$

and see that we can choose $\rho \in \mathbb{R}$ in such a way that (at least) one of the coefficients $\left(\nu_{k}+\rho \tau_{k}\right)$ is zero and the others $\geq 0$. This can be done untill the desired representation is attained.

\section{References}

[1] Avriel, M,Diewert, W.E., Schaible,S. and I.,Zang. Generalized Concavity, volume 36 of Mathematical concepts and Methods in Science and Engineering. Pleneum Press, New York, 1988.

[2] Bazaraa, M.S., Sherali, H.D. and C.M. Shetty. Nonlinear Programming: Theory and Algorithms (second edition). Wiley-Interscience series in Discrete Mathematics and Optimization. Wiley, New York, 1993. 
[3] Bertsekas, D.P. Nonlinear programming. Athena Scientific, Massachusetts, 1995.

[4] Chvátal, V. Linear Programming. W.H Freeman and Company, New York, 1999.

[5] Faigle, U., Kern, K. and G. Still. Algorithmic Principles to Mathematical Programming). Kluwer Academic publishers, Dordrecht, 2002.

[6] Farkas, J. Theorie der einfachen Ungleichungen. Journal für die reine und angewandte Mathematic, 124:1-27, 1902.

[7] John, F. Extremum problems with inequalities as side conditions. In Friedrichs, K.O, Neugebauer, O.E and J.J. Stoker, editor, Studies and Essays, Courant Anniversary Volume. WileyInterscience, 1948.

[8] Karush, W. Minima of functions of several variables with inequalities as side conditions. Master's thesis, Department of Mathematics, University of Chicago, 1939.

[9] Kuhn, H.W and A.W. Tucker. Nonlinear programming. In Neyman, J, editor, Proc. 2nd Berkeley Symposium on Mathematical Statistics and Probability. University of California Press, 1951.

[10] McShane, E.J. The Lagrange multiplier rule. The American Mathematical Monthly, 80(8):922-925, 1973.

[11] Rudin, W. Principles of Mathematical Analysis (third edition). Mc-Graw Hill, New York, 1976. 


\section{Publications in the Report Series Research* in Management}

\section{ERIM Research Program: "Business Processes, Logistics and Information Systems"}

2005

On The Design Of Artificial Stock Markets

Katalin Boer, Arie De Bruin And Uzay Kaymak

ERS-2005-001-LIS

http://hdl.handle.net/1765/1882

Knowledge sharing in an Emerging Network of Practice: The Role of a Knowledge Portal

Peter van Baalen, Jacqueline Bloemhof-Ruwaard, Eric van Heck

ERS-2005-003-LIS

$\underline{\text { http://hdl.handle.net/1765/1906 }}$

A note on the paper Fractional Programming with convex quadratic forms and functions by H.P.Benson J.B.G.Frenk

ERS-2005-004-LIS

$\underline{\text { http://hdl.handle.net/1765/1928 }}$

A note on the dual of an unconstrained (generalized) geometric programming problem

J.B.G.Frenk and G.J.Still

ERS-2005-006-LIS

http://hdl.handle.net/1765/1927

Privacy Metrics And Boundaries

L-F Pau

ERS-2005-013-LIS

http://hdl.handle.net/1765/1935

Privacy Management Contracts And Economics, Using Service Level Agreements (Sla)

L-F Pau

ERS-2005-014-LIS

http://hdl.handle.net/1765/1938

A Modular Agent-Based Environment for Studying Stock Markets

Katalin Boer, Uzay Kaymak and Arie de Bruin

ERS-2005-017-LIS

http://hdl.handle.net/1765/1929

Lagrangian duality, cone convexlike functions

J.B.G. Frenk and G. Kassay

ERS-2005-019-LIS

http://hdl.handle.net/1765/1931

Operations Research in Passenger Railway Transportation

Dennis Huisman, Leo G. Kroon, Ramon M. Lentink and Michiel J.C.M. Vromans

ERS-2005-023-LIS

http://hdl.handle.net/1765/2012

Agent Technology Supports Inter-Organizational Planning in the Port

Hans Moonen, Bastiaan van de Rakt, lan Miller, Jo van Nunen and Jos van Hillegersberg

ERS-2005-027-LIS

http://hdl.handle.net/1765/6636 
Faculty Retention factors at European Business Schools

Lars Moratis, Peter van Baalen, Linda Teunter and Paul Verhaegen

ERS-2005-028-LIS

http://hdl.handle.net/1765/6559

Determining Number of Zones in a Pick-and-pack Orderpicking System

Tho Le-Duc and Rene de Koster

ERS-2005-029-LIS

http://hdl.handle.net/1765/6555

Integration of Environmental Management and SCM

Jacqueline Bloemhof and Jo van Nunen

ERS-2005-030-LIS

http://hdl.handle.net/1765/6556

On Noncooperative Games and Minimax Theory

J.B.G. Frenk and G.Kassay

ERS-2005-036-LIS

http://hdl.handle.net/1765/6558

Optimal Storage Rack Design for a 3-dimensional Compact AS/RS

Tho Le-Duc and René B.M. de Koster

ERS-2005-041-LIS

http://hdl.handle.net/1765/6730

Strategies for Dealing with Drift During Implementation of ERP Systems

P.C. van Fenema and P.J. van Baalen

ERS-2005-043-LIS

http://hdl.handle.net/1765/6769

Modeling Industrial Lot Sizing Problems: A Review

Raf Jans and Zeger Degraeve

ERS-2005-049-LIS

http://hdl.handle.net/1765/6912

Cyclic Railway Timetabling: a Stochastic Optimization Approach

Leo G. Kroon, Rommert Dekker and Michiel J.C.M. Vromans

ERS-2005-051-LIS

http://hdl.handle.net/1765/6957

Linear Parametric Sensitivity Analysis of the Constraint Coefficient Matrix in Linear Programs

Rob A. Zuidwijk

ERS-2005-055-LIS

Diffusion of Mobile Phones in China

Sunanda Sangwan and Louis-Francois Pau

ERS-2005-056-LIS

An Elementary Proof of the Fritz-John and Karush-Kuhn-Tucker Conditions in Nonlinear Programming

S.I. Birbil, J. B. G. Frenk and G. J. Still

ERS-2005-057-LIS

General model for automated diagnosis of business performance

Emiel Caron and Hennie Daniels

ERS-2005-058-LIS 
* A complete overview of the ERIM Report Series Research in Management: https://ep.eur.nl/handle/1765/1

ERIM Research Programs:

LIS Business Processes, Logistics and Information Systems

ORG Organizing for Performance

MKT Marketing

F\&A Finance and Accounting

STR Strategy and Entrepreneurship 